\title{
Comparative Analysis of Various Cloud Technologies
}

\author{
Dr. Gurdev Singh \\ Professor and Head \\ Department of Computer science \& Engineering \\ Adesh Institute of Engineering \& Technology \\ Faridkot (India) \\ singh.gndu@gmail.com
}

\author{
Akanksha \\ Lecturer \\ Department of Computer science \& Engineering \\ Adesh Institute of Engineering \& Technology \\ Faridkot (India) \\ er.akanksha1988@gmail.com
}

\section{ABSTRACT}

With the increasing prevalence and demand of large scale cloud computing environment, a researcher has to draw more attention towards the services provided by the CLOUD. As the access to the server is increasing, centralized and distributed computing architecture will produce bottlenecks data which affect the quality of cloud computing services and bring the huge support to users. In this paper we are going to propose certain vital aspects such as memory utilization, storage capacity to check the efficiency and performance of various clouds in cloud computing environment. This is based upon the static data. The proposed mechanism enables users to access memories in various systems depending on the predefined criteria. Selection method for accessing the memory of a resource is properly introduced in this paper. Our evaluation results show that the aggregation of various clouds is effective in indicating the better efficiency and also to reduce network traffic sent over cloud networks.

\section{General Terms}

Cloud computing, Platform as a service, Performance, Software as a service, Distributed computing

\section{Keywords}

WAC, AC, UEC, EC2, SaaS, GAE

\section{INTRODUCTION}

Cloud computing is a promising next-generation computing epitome which primarily relies on technologies such as concurrency, consistency, stability, scalability, validity, transparency and so forth. Cloud services, which are deployed as self-contained components, are normally partial solutions that must be composed to provide a single virtualized service to Cloud [1]. Those famous companies including Amazon, IBM, HP, Google and Microsoft are creating and deploying Clouds in various locations around the world [2].

Data and information related to different users and applications can be processed and stored in cloud computing environments to provide favorable deed. The benefits from cloud computing include significant reduction of upfront cost, increased utilization through sharing of physical resources, ease of resource management, flexibility and elasticity of provisioning resources, ease of programmability and management of

distributed applications [3]. Cloud deals with the exchange of data and information among various databases, networks and systems through servers by using any of the LAN, MAN and
WAN networking. The interlinking and connection between various sources is shown below:

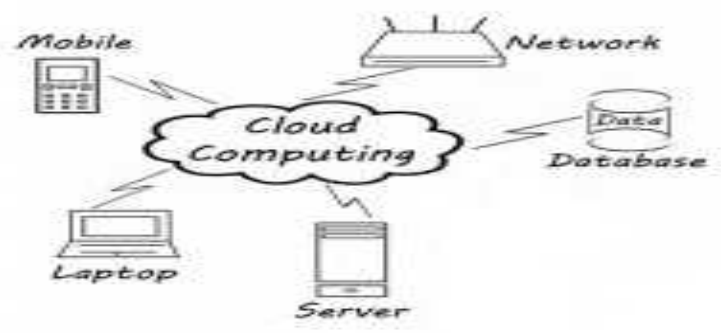

Figure 1: Cloud Computing

With this platform, companies will have the prospect to dynamically scale their hardware and software prerequisites in a more efficient way.

To further idealize the concept, firstly discuss the various Clouds providing platforms along with their specifications, usability and analyze their impact on itself and on other Clouds or vice-versa.

\subsection{Various Cloud Providers}

In the past years, cloud computing gained rapid development on a worldwide scale. First, it had been widely accepted by the IT industry, and frequently completed the changes from the technical concept to business model. For example Microsoft has launched the "Windows Azure" program, IBM launched "Blue Cloud" program, and Amazon has launched Amazon Elastic Compute Cloud (EC2) is a web service that provides resizable compute capacity in the cloud. It is designed to make web-scale computing easier for developers [4]. Google App Engine opens the cloud computing platform to the users who can trusteeship application on Cloud platform and enjoy certain applications for free.

There are number of (approximate 90 in the world) Cloud Companies such as Amazon, Google Apps, Equinix, Eucalyptus, Red Hat, Okta, Microsoft, VMWare, Rackspace, Savvis, Caspio, Bluewolf, LayeredTech, Voxeo, Cloud Switch, Nubifer, Cordys, Tropo, Cloudera, Clustercorp etc, that are works on cloud platform to make business innovations. They seem to be look like this:

\section{Choose Cloud Provider:}




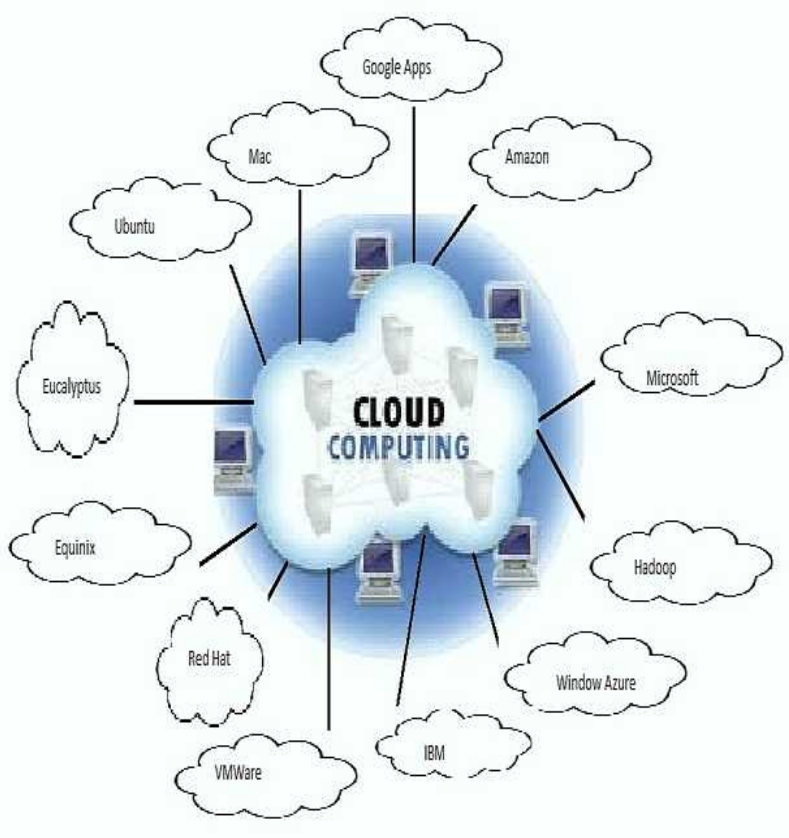

Figure 2: Cloud Provider

But here we discuss few of them.

\subsubsection{Google Cloud}

Google has been pushing the technological bounds of cloud computing for more than ten years [5]. Apart from the highly popular Google Search, Gmail, Chrome browser and Android OS there are various Cloud services from Google. Most of these services can be categorized under SaaS (Software as a Service) and are free. It referred to as "on-demand software. A few, like Google Maps, are free only for non-commercial use [6].

Google Cloud provides lots of services such as Google Public DNS, Google Profile, Google +1, Google Docs, Google Cloud Print, iGoogle, Blogger, Google App Engine (GAE) and many more.GAE used more in these days. It is explain as below:

Google App Engine applications are easy to build, easy to maintain, and easy to scale as your traffic and data storage needs grow. With App Engine, there are no servers to maintain: You just upload your application, and it's ready to serve your users [7]. GAE provides a powerful distributed data storage service that features a query engine and transactions. The datastore stores objects or entities instead of rows or records. It is strongly consistent and uses optimistic concurrency control. Now these days Google Docs is more preferable. User use Google docs to work on files anywhere and anytime. This is SaaS based. SaaS applications are often updated more frequently than traditional software. It helps in providing faster, and real-time, collaboration as it can be accessed from any connected device. Along with this it quickly analyzes and organizes data.

GAE related with certain implicit requirements for describing and identifying the app completely .

\section{i) C.P.U. Capacity: 1GHz-16GHz}

ii) Memory Utilization: 1GB-10 GB iii) Instance Space: $128 \mathrm{MB}-1 \mathrm{~GB}$

iv) Language Support: Java, Python

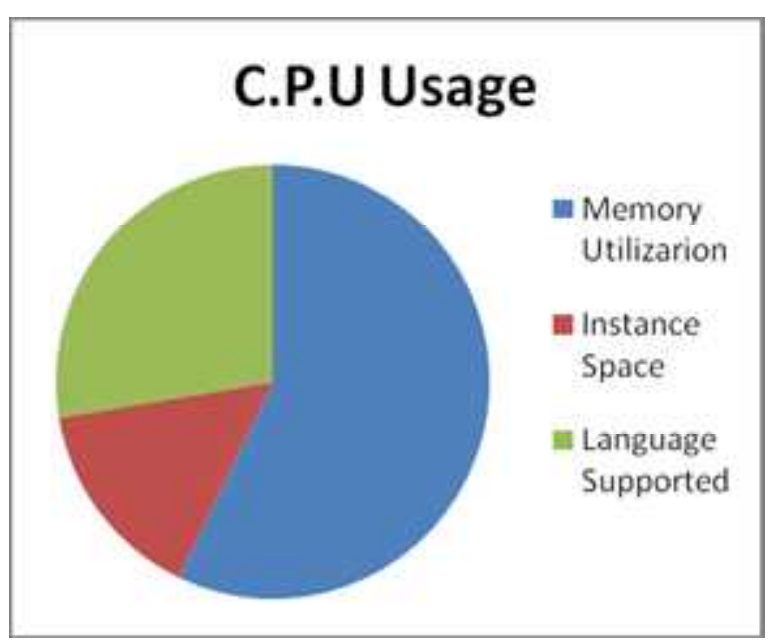

Figure 3: CPU1

It provides $1 \mathrm{~GB}$ of free storage space for data and information storage.

\subsubsection{Windows Azure Cloud (WAC)}

Windows Azure Cloud is launched by Microsoft Corporations. Today Cloud Computing is a really overloaded term then SOA [8]. SOA is Service Oriented Architecture. A service-oriented architecture is essentially a collection of services. These services communicate with each other. The communication can involve either simple data transmission or it could involve two or more services coordinating some action.

The following figure illustrates it:

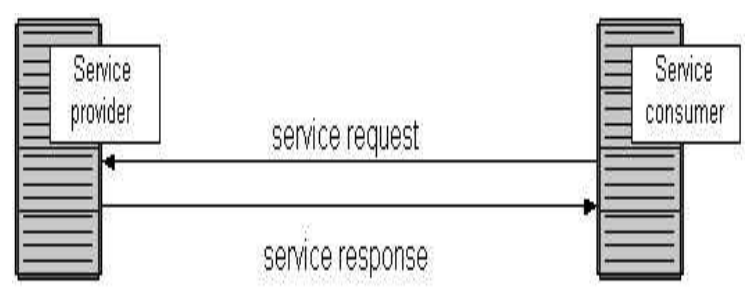

Figure 4 : Cloud

It is a basic service-oriented architecture. It shows a service consumer at the right side sending a service request message to a service provider at the left side. The service provider returns a response message to the service consumer. The request and subsequent response connections are defined in such a manner that it is understandable to both the service consumer and service provider.

Microsoft's cloud includes SaaS (Software-as-a-Service) offerings as shown in the top row of the below diagram, such as Windows Live and the Business Productivity Online Suite and the PaaS (Platform-as-a-Service) offering currently branded as the Azure Services Platform [8]. 


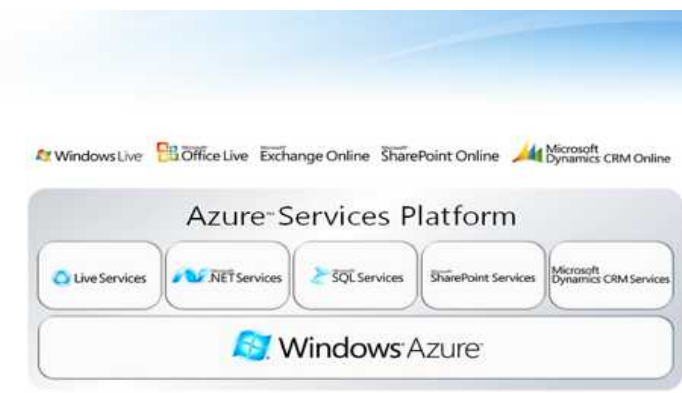

Figure 5: Cloud 2

It represents application containers that perform at a higher level in the stack, instead of Windows Server VM (Virtual Machines) instances for user to use directly. In fact, it actually required more engineering effort, but the final result is that platform which provides extreme scalability, availability, the transparency of highly distributed and replicated processes and data, while hiding the complexities of the systems automation and management operations on a network of globally distributed data sources. It deals with GC (Gladinet Cloud) which is further subdivided into two parts.

a) GCD ( Gladinet Cloud Desktop) access cloud storage like a local drive and backup of files, folders and email to any cloud storage service. It sync data across multiple computers using any cloud storage and secure your cloud storage with military grade encryption [9] .

b) GCS ( Gladinet Cloud Server) attach Cloud storage to your file server and protect your file, database and mail server with cloud backup It access cloud storage using CIFS/NFS with AD integration and securely access your file server anywhere with GC [9].

WAC relates with certain aspects of implicit requirements and specifications which are refer as below:

\section{i) C.P.U. Capacity: $1 \mathrm{GHz}-8 * 1.6 \mathrm{GHz}$}

ii) Memory Utilization: 768MB-14GB

iii) Instance S pace: 20GB-2040 GB

iv) Language Support: VB.NET, C\#, PHP

\subsubsection{Amazon Cloud (AC)}

Amazon Coud is describe by using certain set of instances may be little, small orlarge. The amount of CPU that is allocated to a particular instance is expressed in terms of these S3 and EC2 Compute Units. Amazon S3 is storage for the Internet. Data stored in Amazon S3 is secure by default. Amazon Elastic Compute Coud (Amazon EC2) is a web service that provides resizable compute capacity in the doud. It deals with certain set of services such as elasticity, flexibility, reliability, auto scaling etc. EC2 and S3 are designed to make web-scale computing easier for developers [10].

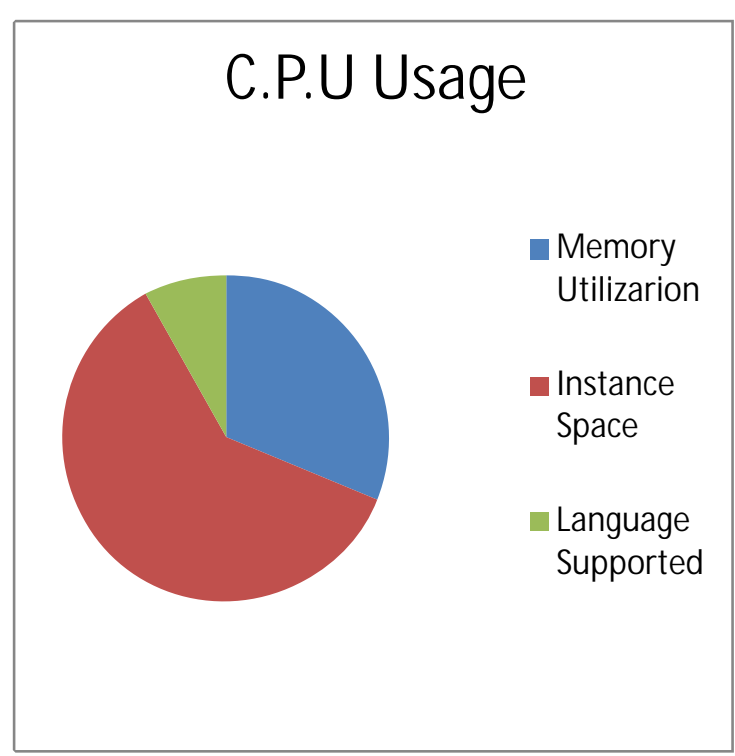

Figure 6: CPU2

Amazon Cloud is refers to ACD (Amazon Cloud Drive). ACD defines as your personal hard drive in the cloud and stores music, videos, photos, and other documents on Amazon's secure servers. The files stored in ACD are secure even the computer crashes, or is lost or stolen.

Amazon Cloud based upon straining in a cloud. For better performance path-finder window option is generated for faster pulling of songs and albums for uploading and naming the files.

This AC depends upon explicit set of specifications:

i) C.P.U. Capacity: $1 \mathrm{GHz}-20 * 3.2 \mathrm{GHz}$

ii) Memory Utilization: 1.7 GB-7GB

iii) Instance Space: 160GB-1690GB

iv) Language Support: Any

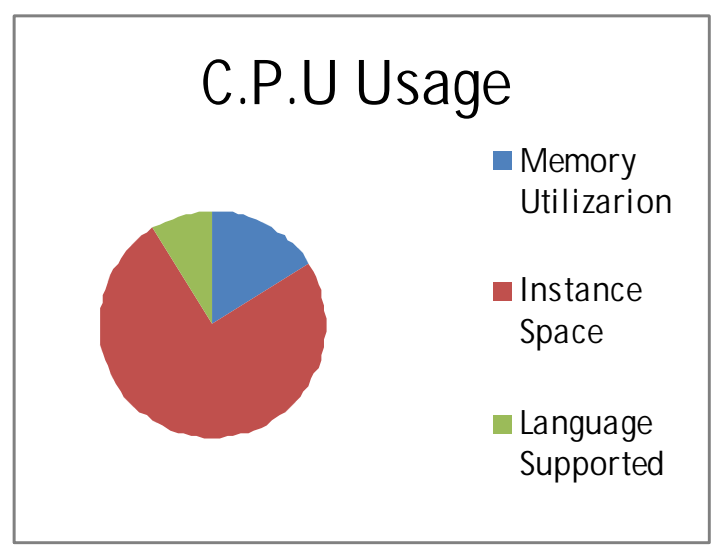

Figure 7: CPU3

It provides $5 \mathrm{~GB}$ of free storage space. 


\subsubsection{Ubuntu Enterprise Cloud}

UEC is design to lean against a de-facto standard (Amazon EC2) to offer and open source implementation (Eucalyptus) as a foundation on which we will offer an integration of best of breed Open Source tools [11]. Eucalyptus works on IaaS (Infrastructure as a Service) style private and hybrid clouds. Designing for EC2 assures on-demand deployment and scalability, two key benefits of cloud computing. BUT requires new appliance architecture.UEC is perfect match for EC2.EC2 is public cloud and UEC is private cloud.

Ubuntu will help you to construct your own cloud for internal usage or like a cloud support for other users. Products and solutions, such as Ubuntu Server, are completely optimized to operate on both private and public cloud infrastructures.

Ubuntu Cloud offers immediacy and elasticity in your IT infrastructure.

The various aspects of UEC refers as :

i) C.P.U. Capacity: $1 \mathrm{GHz}-2 * 2 \mathrm{GHz}$

ii) Memory Utilization: 512 MB-2GB

iii) Instance S pace: 40GB-200GB

iv) Language Support: Linux

\section{COMPARISON BETWEEN VARIOUS CLOUDS}

Cloud computing is a new and rapidly growing technology, allowing user to use applications or store files through the internet without having to store them on the physical computer. On the basis of above mention data and information the comparison of various Clouds are to be considered.

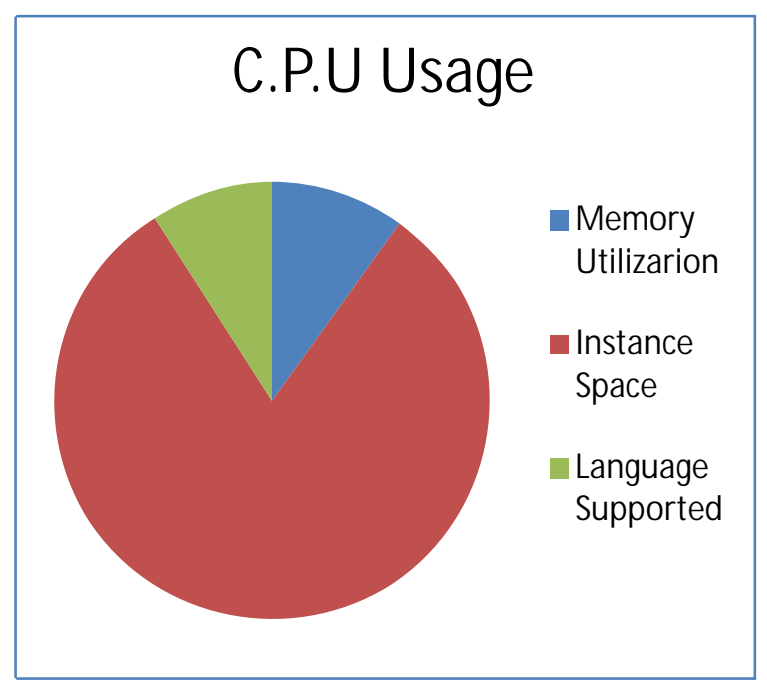

Figure 8: CPU4

This is to be shown by taking their Input/output performance which is shown below graphically:

\subsection{Input/Output Pe rformance}

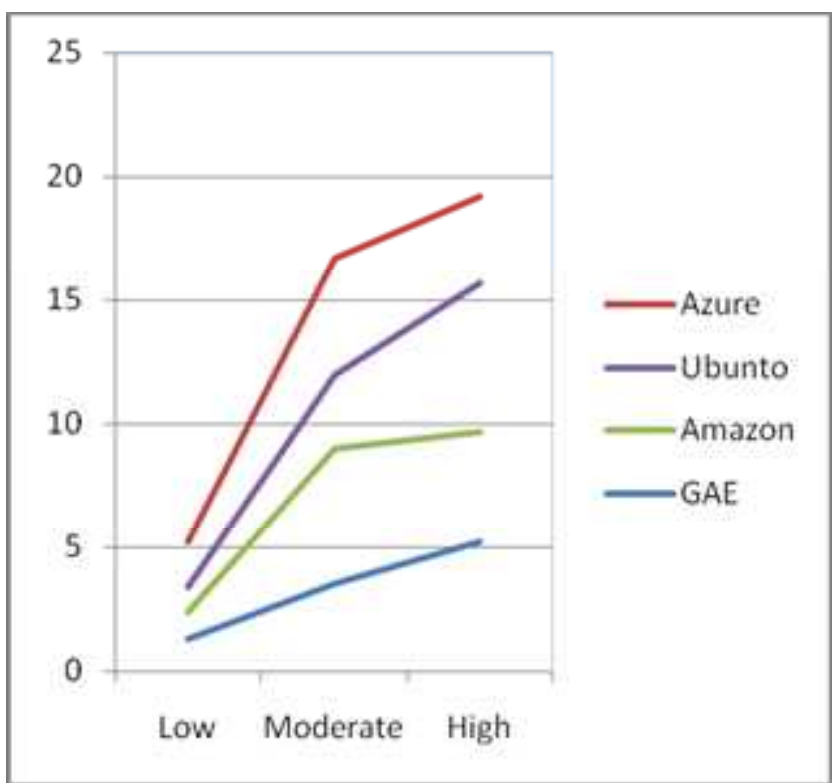

Figure 7: Analysis

This graph indicates that the performance of GAE is start from low level and moves towards high level on the basis of their specifications. The Amazon cloud indicates the flow from moderate level to first high level than to moderate level and again final level. This shows the variation of functionality perform by it.WAC relies the path start from lower level to moderate and finally shows its performance to peak level. Last but not the least the Ubuntu Cloud describes the flow in the positive sense means moderate to high level.

\section{CONCLUSION}

Cloud computing relies on the internet and gives users the ability to connect from anywhere. In order to analyses the various clouds, it is necessary to support the theoretical validation of the study with practical validation. In this paper we did the same. We concentrate on theoretical part because a practical part is completely useless without it. While the experiments run in the cloud were neither exhaustive nor complex, they provided a step towards understanding the cloud better, especially in terms of the CPU Usage and its Performance.

\section{REFERENCES}

[1] "J. Octavio Gutierrez-Garcia, Kwang-Mong Sim", "SelfOrganizing Agents for Service Composition in Cloud Computing" 2nd IEEE International Conference on Cloud Computing Technology and Science

[2] R. Buyya, "Market-Oriented Cloud Computing: Vision, Hype, and Reality of Delivering Computing as the 5th Utility", Proc. of $9^{\text {th }}$ IEEE/ACM International Symposium on Cluster Computing and the Grid (CCGRID'09), Shanghai, China, May, 2009, pp. 1, doi:10.1109/CCGRID.2009.97. 
[3] "M. Suhail Rehman, Majd F. Sakr",Initial Findings for Provisioning Variation in Cloud Computing, 2nd IEEE International Conference on Cloud Computing Technology and Science.

[4] “Amazon Elastic Compute Cloud (Amazon EC2) "http://aws.amazon.com/ec2/

[5] "Advantages of Google's cloud - Google Apps”www.google.com/apps/intl/en/business/ cloud.html

[6] "30 + Google Cloud Computing Services for You |Cloud Computing" www.techno-pulse.com/2011/.../google-cloudcomputing-services.html

[7] "What Is Google App Engine?-Google

Code"code.google.com/appengine/docs/whatisgoogleap pengine. html

[8] "Cloud Computing and the Microsoft Platform-Architecture + Strategy -Site Home-MSDN Blogs"
[9] "GLADINET - Cloud Storage Access Platform \& Solutions.html.

[10] "Amazon Web Services (Amazon S3, Amazon EC2).htm"

[11]"nicolas.barcet.com/drupal/.../SkillsMatter/WhatIsUbuntuC loud_0.pdf” 